\title{
FACTORES PREDICTORES DE USO PROBLEMÁTICO DE ALCOHOL EN PERSONAS ATENDIDAS EN UNA SALA DE EMERGENCIA
}

\author{
Fabián Fiestas ${ }^{1, a}$, Javier Ponce ${ }^{1, b}$, Carla Gallo ${ }^{1, c}$, Inés Bustamante ${ }^{2, d}$, \\ Carlos Ordóñez ${ }^{3, \mathrm{e}}$, Guido Mazzotti1 ${ }^{1,4, \mathrm{e}}$.
}

\begin{abstract}
RESUMEN
Objetivos. Valorar el efecto predictivo de características claves de pacientes atendidos en salas de emergencia para detectar casos de uso problemático de alcohol. Materiales y Métodos. La muestra de estudio estuvo constituida por 371 personas atendidas en el lapso de siete días completos de enero de 2005 en el servicio de emergencia de un hospital público de Lima, Perú. Se aplicó un cuestionario demográfico, el SIDUC/CICAD para uso reciente de sustancias psicoactivas en salas de emergencias (i.e., uso dentro de las seis horas previas a la atención) y el AUDIT para uso problemático de alcohol en el último año. El análisis de regresión logística simple y multivariada permitió valorar el efecto predictor de la edad, sexo, especialidad del servicio de atención, presencia de daño físico y el uso reciente de alcohol para detectar casos problemáticos de su uso. Resultados. El odds de tener uso problemático de alcohol en los varones es 26 veces el odds de tener dicho problema entre las mujeres $(p<0,001)$. El análisis estratificado por sexo y ajustado por edad y especialidad del servicio de emergencia, mostró que para los varones el uso reciente de alcohol estuvo fuertemente asociado con presentar uso problemático (OR=5,2; IC al 95\%: 2,4-11,5; $p<0,001)$, mientras que para las mujeres no se encontró dicha asociación. Conclusiones. Se debe tamizar uso problemático de alcohol en aquellos varones con uso reciente de alcohol atendidos en salas de emergencia, para poder identificarlos y darles la oportunidad de recibir consejería o un manejo médico adecuado.
\end{abstract}

Palabras clave: Abuso de alcohol, Atención de Emergencia, Predicción (fuente: DeCS BIREME).

\section{PREDICTIVE FACTORS OF ALCOHOL USE PROBLEMS AMONG PATIENTS VISITING AN EMERGENCY ROOM}

\begin{abstract}
Objectives. To assess the predictive effect of key individual-level characteristics to identify cases of alcohol use problems among patients visiting an emergency room. Materials and methods. The study sample was composed of 371 people attending an emergency room in a public hospital in Lima, Peru, during a period of seven complete days in January, 2005. For data gathering, we used a questionnaire for demographic information, the SIDUC/CICAD for recent use (i.e., in the last 6 hours) of psychoactive substances before arriving to the emergency room, and the AUDIT, to identify alcohol use problems in the last year. Univariate and multivariate logistic regression models were used to estimate the predictive effect of age, sex, area of attention in the emergency room, presence of physical injuries and recent use of alcohol. Results. The odds of being a case of alcohol use problem for males is 26 times the odds of having that problem for females $(p$-value $<0,001)$. Stratified analysis by sex and adjusted by age and area of attention in the emergency room, showed that males who had recent use of alcohol were more likely of having alcohol use problems as compared to those who did not have recent alcohol use (OR=5.2; 95\% Cl: 2.4 - 11.5; $p<0,001)$, while for females such an association did not exist. Conclusions. These results support screening initiatives for those males who have recent alcohol use before arriving to an emergency room in order to identify cases of alcohol-related problems and refer them for an appropriate counseling or medical treatment.
\end{abstract}

Key Words: Alcohol Abuse, Emergency Attendance, Forecasting (source: MeSH NLM).

\section{INTRODUCCIÓN}

El alcohol se posiciona entre los factores que más pérdidas económicas generan a las sociedades (1), además del sufrimiento que significa a individuos y familias (2). En el mundo, el uso de alcohol explica aproximadamente el $4 \%$ de años perdidos por discapacidad o muerte prematura, lo cual coloca a esta sustancia entre las cinco primeras causas de carga de enfermedad ${ }^{(3)}$, siendo especialmente problemático para los varones entre 15 y 44 años ${ }^{(4)}$. En el Perú, el abuso y la dependencia al alcohol figuran como la tercera

\footnotetext{
1 Laboratorios de Investigación y Desarrollo, Facultad de Ciencias y Filosofía, Universidad Peruana Cayetano Heredia, Lima, Perú.

2 Facultad de Salud Pública y Administración Carlos Vidal Layseca, Universidad Peruana Cayetano Heredia, Lima, Perú.

3 Jefe del Departamento de Adicciones, Hospital Hermilio Valdizán, Lima, Perú.

4 Facultad de Medicina Alberto Hurtado. Universidad Peruana Cayetano Heredia, Lima, Perú.

+ Fallecido.

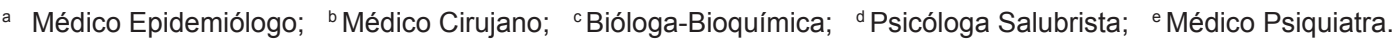

Recibido: 16-11-10 Aprobado: 09-03-11 
causa de carga de enfermedad, superados solo por la neumonía y los accidentes de tránsito ${ }^{(5)}$. Sin embargo, la contribución del uso de alcohol puede ser mayor si tomamos en cuenta que este tiene asociación causal con una variedad de otros problemas de salud, como accidentes de tránsito, violencia, accidentes caseros y de trabajo, uso de drogas ilegales o involucramiento en conducta sexual de riesgo ${ }^{(6-16)}$, sin que necesariamente medie la existencia de abuso o dependencia en dichas asociaciones.

A pesar que el uso problemático de alcohol es una prioridad de salud, aún muchas personas con dichos problemas permanecen no diagnosticadas, sin tratamiento o con tratamientos inadecuados. Por ejemplo, para Latinoamérica y el Caribe se ha estimado que aproximadamente tres de cada cuatro personas con abuso o dependencia al alcohol no reciben tratamiento alguno en servicios de salud especializados o generales ${ }^{(17)}$. Un contexto similar puede estar dándose para Perú, donde se ha reportado que hay poca demanda satisfecha de tratamiento para desórdenes mentales ${ }^{(18-20)}$.

Muchos factores pueden explicar la gran proporción de personas con problemas de alcohol que no reciben tratamiento. Entre los factores relacionados con los sistemas de salud se puede mencionar, por ejemplo, no sólo la escasez de instituciones y profesionales de la salud especializados en el tratamiento de problemas de abuso o dependencia de alcohol, sino también al olvido en que las instituciones de salud en general colocan a este tipo de problemas. A ellos se añade el descuido crítico en que se encuentra la salud mental en nuestras sociedades, que lleva a que muy pocos recursos financieros y logísticos en instituciones públicas sean orientados a cubrir el tratamiento de enfermedades mentales y problemas por uso de sustancias, que, sumándose a la ausencia de aseguramiento de salud por estos problemas, da como resultado que muchas personas tengan grandes dificultades para acceder a tratamientos óptimos. A nivel individual, podemos mencionar el desconocimiento, el estigma y la creencia que tienen las personas y miembros de las familias que los problemas de uso de alcohol no son problemas de salud y que, por lo tanto, no requieren ayuda médica y que más bien dependen de la voluntad del individuo, a quien incluso pueden culpar de tener dichos problemas.

En el contexto de pocos recursos en el sistema de salud y poca necesidad de tratamiento percibida, acciones implementadas en salas de emergencia pueden ser pasos iniciales para incrementar a bajo costo y en el corto-mediano plazo el número de personas en tratamiento por problemas de uso de alcohol. El alcohol en pacientes atendidos en las salas de emergencia es frecuente ${ }^{(21)}$, tanto en la forma de intoxicación aguda ${ }^{(6)}$, como en la forma de uso crónico ${ }^{(22)}$, independientemente de la razón por la que el individuo acudió al servicio de emergencia. En el Perú, nuestro grupo encontró que alrededor del $40 \%$ de varones y $3 \%$ de mujeres que habían sido atendidos en el servicio de emergencia de un hospital general en Lima, Perú, alcanzaron una puntuación de ocho o más en el Alcohol Use Disorders Identification Test (AUDIT), puntaje que sugiere la presencia de algún tipo de uso problemático de alcohol en el último año, incluyendo dependencia ${ }^{(23)}$.

El hecho de que haya una concentración importante de personas con un uso problemático de alcohol en las salas de emergencia, hace de este tipo de servicio hospitalario un punto clave en la captación, manejo inicial y derivación hacia tratamiento especializado de personas con dichos problemas, como ha sido la experiencia con las intervenciones breves ${ }^{(24)}$. No obstante esta oportunidad que ofrecen los servicios de emergencia, las demandas del trabajo que las enfermedades que se atienden en estos servicios supone, más la escasez de personal, hace que un trabajo de tamizaje masivo con instrumentos ad hoc entre las personas atendidas, resulte siendo poco factible. Además, debido a que no todos los pacientes en las salas de emergencia tienen uso problemático de alcohol, es necesario contar con métodos capaces de seleccionar a aquellas personas con alto riesgo de ser casos positivos para así orientar de manera costo-efectiva los esfuerzos de tamizaje más extensos para identificar personas con dichos problemas.

El presente estudio tiene el objetivo de identificar características que sean predictivas de la presencia de uso problemático de alcohol entre aquellas que son fácilmente identificadas a partir de la observación simple o por medio de preguntas sencillas por el personal de salud al momento de recibir a un paciente en la sala de emergencia (como son el sexo, la edad, la especialidad del servicio en la que se recibió la atención de emergencia, presencia de daño físico y el uso de alcohol en las seis horas previas a la atención en el servicio de emergencia).

\section{MATERIALES Y MÉTODOS}

\section{PARTICIPANTES}

La población de estudio (i.e., a quienes podrán ser extrapolados los resultados de este estudio) es aquella atendida en el Servicio de Emergencia del Hospital Nacional Cayetano Heredia, el cual es un hospital general ubicado en la zona norte de la ciudad de Lima, 
Perú, con un área de influencia de cerca de tres millones de personas. La muestra del estudio estuvo conformada por todas aquellas personas de 14 años de edad o más, de ambos sexos, atendidos en el lapso de siete días del mes de enero del año 2005, incluyendo las 24 horas de cada día, en los servicios de gínecoobstetricia, medicina y cirugía en el Departamento de Emergencia de dicho hospital. No se incluyeron personas atendidas en las unidades de cuidados intensivos, trauma-shock o de reanimación. Debido a que un gran porcentaje de atenciones en el servicio de gínecoobstetricia fueron mujeres en trabajo de parto y puesto que muy pocas de las pacientes de este servicio reportaron uso reciente de alcohol y aun un menor número de ellas reportaron problemas de uso crónico de esta sustancia, para el objetivo del presente artículo serán excluidas del análisis todas las mujeres atendidas en este servicio.

Se registraron 460 atenciones en los servicios de cirugía y medicina durante el tiempo de realización del estudio. El nivel de participación en el estudio fue de aproximadamente $80 \%$, lo que significa que 93 de las 460 personas atendidas no aceptaron participar. Aunque no hubo diferencias en los niveles de rechazo por servicio ni edad, sí hubo una mayor probabilidad entre las mujeres de no participar (Tabla 1). Respecto a las razones de no participación, el 30\% fue porque no dieron su consentimiento y el resto fue debido a limitaciones logísticas del equipo de reclutamiento de invitar al paciente al estudio por motivos de paso rápido a otros servicios del hospital (p.ej. paso a sala de operaciones), deterioro clínico, fuga o sobredemanda.

Tabla 1. Características demográficas asociadas a la participación durante el tiempo de implementación del estudio en los servicios de cirugía y medicina del Departamento de Emergencia.

\begin{tabular}{|c|c|c|c|}
\hline Variable & $\begin{array}{c}\text { Aceptó } \\
\text { participar n (\%) }\end{array}$ & $\begin{array}{c}\text { No aceptó } \\
\text { participar } n(\%)\end{array}$ & $\begin{array}{c}\text { Valor } \\
\mathbf{p}\end{array}$ \\
\hline \multicolumn{4}{|l|}{ Sexo } \\
\hline Masculino & $200(84,8)$ & $36(15,2)$ & \\
\hline Femenino & $155(73,5)$ & $56(26,4)$ & $0,003^{*}$ \\
\hline Total† & $355(79,4)$ & $92(20,6)$ & \\
\hline \multicolumn{4}{|l|}{ Servicio } \\
\hline Medicina & $224(77,2)$ & $66(22,8)$ & \\
\hline Cirugía & $143(84,1)$ & $27(15,9)$ & $0,08^{*}$ \\
\hline Total & $367(79,8)$ & $93(20,2)$ & \\
\hline $\begin{array}{l}\text { Edad promedio } \\
\text { (desviación } \\
\text { estándar) }\end{array}$ & $36,3(17,2)$ & $38,8(23,3)$ & $0,29 \ddagger$ \\
\hline
\end{tabular}

\section{ASPECTOS ÉTICOS}

El estudio fue aprobado por los Comités Institucionales de Ética de la Universidad Peruana Cayetano Heredia y del Hospital Nacional Cayetano Heredia. Los participantes fueron informados del proyecto y dieron su consentimiento voluntario para participar en el estudio. En el caso de menores de edad, se solicitó tanto el consentimiento de uno de los padres o un apoderado como el asentimiento del propio menor.

\section{INSTRUMENTOS}

Se usaron tres instrumentos en este estudio. El primero correspondió a un cuestionario demográfico que incluyó, además de edad, sexo, estado civil y nivel educativo, preguntas acerca de la especialidad del servicio en que se atendió al paciente, el motivo de la atención, clasificación de las heridas en caso de daño físico, circunstancias y el lugar en que se produjo el daño físico. Este instrumento fue validado, en cuanto a contenido, mediante el método de jueces a cargo de profesionales con experiencia en investigación tanto en salud mental y drogas como en medicina de emergencias.

El segundo instrumento aplicado fue la Encuesta de Pacientes en Salas de Urgencia desarrollada por el Sistema Interamericano de Datos Uniformes sobre Consumo de Drogas (SIDUC) de la Comisión Interamericana para el Control del Abuso de Drogas (CICAD), en el que se registra el motivo de ingreso a la sala de emergencia, el estado de conciencia y el consumo de sustancias, incluyendo drogas ilegales (p.ej. cocaína, marihuana), inhalantes, drogas de prescripción médica (p.ej. sedantes y tranquilizantes) y el alcohol, en las seis horas previas a la atención de emergencia, según el proveedor o paciente ${ }^{(25)}$. Finalmente, para la identificación de usuarios con consumo problemático de alcohol se usó el Alcohol Use Disorder Identification Test (AUDIT)(26), el cual ha sido validado al idioma español por Rubio et al. ${ }^{(27)}$. Este instrumento presenta una sensibilidad del $80 \%$ y especificidad del $90 \%$ para el diagnóstico de uso problemático de alcohol (26-29). Específicamente, un puntaje de 0 a 7 es catalogado como "consumo sin riesgo" de alcohol; de 8 a 15 es "consumo de riesgo"; de 16 a 19 es "consumo perjudicial" y de 20 o más es "dependencia probable" (28). El Alfa de Cronbach del AUDIT para la población del presente estudio fue de 0,89 .

\section{PROCEDIMIENTOS}

La recolección de datos se siguió cubriendo las 24 horas de cada día en turnos de 12 horas durante 7 días. En cada turno participaron tres entrevistadores, distribuidos 
en cada uno de los servicios de emergencia antes mencionados. En los días en los que se esperaba mayor afluencia de pacientes (viernes, sábado y domingo) se requirieron dos entrevistadores adicionales, totalizando cinco entrevistadores por turno. El perfil de los entrevistadores fue el siguiente: trece entrevistadores fueron médicos recién graduados y uno fue un estudiante de medicina del quinto año. Los entrevistadores fueron capacitados durante tres días para garantizar la calidad de la recolección de datos.

\section{ANÁLISIS ESTADÍSTICO}

En el análisis descriptivo, para las variables categóricas se calcularon porcentajes con sus respectivos intervalos de confianza al $95 \%$. Para las variables continuas se realizaron análisis de tendencia central (media, mediana, desviación estándar y percentiles). Los análisis de asociación bivariada se calcularon usando chi cuadrado, test de McNemar y $t$ de Student según corresponda. El análisis para valorar la asociación independiente de los posibles predictores (sexo, edad, uso de alcohol en las seis horas previas a la atención en el servicio de emergencia, presencia de daño físico y el servicio en el que se recibió la atención) con el uso problemático de alcohol (definido por un AUDIT mayor a siete) se realizó utilizando la técnica de regresión logística múltiple. Se consideró como significativo un valor de $p<0,05$. Los análisis fueron hechos en el paquete estadístico STATA $9,0^{(30)}$.

\section{RESULTADOS}

De los 371 participantes en el presente estudio, 224 fueron atendidos en el servicio de medicina y 143 en el de cirugía (cuatro personas no tuvieron registro del servicio al que llegaron). Respecto al sexo de los participantes, 200 fueron varones y 159 mujeres (12 personas no tuvieron registro de sexo). Los varones estuvieron sobrerrepresentados en el servicio de cirugía (varones, $73 \%$ y mujeres, $27 \%$ ), mientras que las mujeres lo estuvieron en el servicio de medicina (varones $46 \%$, mujeres $54 \%$; $p<0,001$ ). Los casados y convivientes representaron aproximadamente el $50 \%$ de los participantes, mientras que los solteros el $40 \%$; y el $65 \%$ había completado la secundaria.

La Tabla 2 muestra la distribución de los participantes respecto a las variables de interés de este estudio por categoría de uso de alcohol. Así, los participantes que puntuaron en el AUDIT en las categorías de uso de alcohol de riesgo, uso perjudicial y dependencia probable, tuvieron mayor probabilidad de ser atendidos en el servicio de cirugía que en el de medicina $(p<0,001)$. Es interesante notar que cerca del $8 \%$ de las personas que fueron atendidas en el servicio de cirugía, estuvieron cataloga-

Tabla 2: Distribución de características demográficas y clínicas por categorías de uso crónico de alcohol en el último año, definidas por el AUDIT.

\begin{tabular}{|c|c|c|c|c|c|c|}
\hline \multirow[b]{2}{*}{ Variable } & \multicolumn{6}{|c|}{ Tipo de uso crónico de alcohol según categorías del AUDIT } \\
\hline & $\mathbf{N}$ & $\begin{array}{c}\text { Sin riesgo* } \\
n(\%)\end{array}$ & $\begin{array}{c}\text { De riesgo } \\
\mathrm{n}(\%)\end{array}$ & $\begin{array}{c}\text { Perjudicial } \\
\text { n (\%) }\end{array}$ & $\begin{array}{c}\text { Dependencia } \\
\mathrm{n}(\%)\end{array}$ & Valor $\mathrm{p}$ \\
\hline Servicio & & & & & & $<0,001$ \\
\hline Medicina & 224 & $184(82,1)$ & $35(15,6)$ & $3(1,3)$ & $2(0,9)$ & \\
\hline Cirugía & 143 & $97(67,3)$ & $26(18,2)$ & $9(6,3)$ & $11(7,7)$ & \\
\hline Sexo & & & & & & $<0,001$ \\
\hline Femenino & 159 & $155(97,5)$ & $4(2,5)$ & 0 & 0 & \\
\hline Masculino & 200 & $119(59,5)$ & $57(28,5)$ & $11(5,5)$ & $13(6,5)$ & \\
\hline Edad & & & & & & 0,07 \\
\hline De 14 a 18 & 41 & $31(75,6)$ & $9(22)$ & $1(2,4)$ & 0 & \\
\hline De 19 a 25 & 86 & $60(69,8)$ & $17(19,8)$ & $4(4,6)$ & $5(5,8)$ & \\
\hline De 26 a 34 & 83 & $61(73,5)$ & $17(20,5)$ & $4(4,8)$ & $1(1,2)$ & \\
\hline De 35 a 44 & 55 & $39(70,9)$ & $10(18,2)$ & $3(5,4)$ & $3(5,5)$ & \\
\hline De 45 a 64 & 72 & $61(84,7)$ & $7(9,7)$ & 0 & $4(5,6)$ & \\
\hline 65 o mayor & 34 & $33(97,1)$ & $1(2,9)$ & 0 & 0 & \\
\hline Alcohol en últimas seis horas & & & & & & $<0,001$ \\
\hline No & 305 & $251(82,3)$ & $42(13,8)$ & $7(2,3)$ & $5(1,6)$ & \\
\hline Sí & 66 & $34(51,5)$ & $19(28,8)$ & $5(7,6)$ & $8(12,1)$ & \\
\hline \multicolumn{6}{|c|}{ Daño físico en pacientes atendidos en cirugía } & 0,10 \\
\hline No & 23 & $19(82,6)$ & 0 & $2(8,7)$ & $2(8,7)$ & \\
\hline Sí & 120 & $78(65)$ & $26(21,7)$ & $7(5,8)$ & $9(7,5)$ & \\
\hline
\end{tabular}

* Incluye a aquellos que han sido abstemios durante el último año 
das como posibles dependientes al alcohol por el AUDIT. En cuanto a la distribución por sexo, aproximadamente $40 \%$ de los varones presentaron algún tipo de consumo problemático de alcohol, mientras que solo $3 \%$ de las mujeres presentaron este problema $(p<0,001)$. Todos los casos de mujeres con uso problemático de alcohol $(n=4)$ fueron por uso de riesgo y ninguno por uso perjudicial o de dependencia probable. La edad no estuvo estadísticamente asociada con las categorías de consumo problemático de alcohol, aunque cabe resaltar que cerca de un $25 \%$ de las personas de entre 14 y 18 años presentan ya algún tipo de problema de uso de alcohol.

La Tabla 2 también muestra que el uso de alcohol en las seis horas previas a la atención en la sala de emergencia (uso reciente) estuvo fuertemente asociado con la presencia de algún tipo de consumo problemático de alcohol en el análisis bivariado. Específicamente, alrededor de $50 \%$ de los que tuvieron uso reciente de alcohol tuvieron algún problema con el alcohol frente al $18 \%$ de los que no tenían uso reciente de dicha sustancia previo a la atención en la sala de emergencia. El 12\% de usuarios recientes de alcohol fueran clasificados como probables dependientes al alcohol. Además, en la misma tabla se observa que el daño físico en las personas atendidas en el servicio de cirugía no estuvo asociado con uso problemático de alcohol.

La Tabla 3 muestra los odds ratios (OR), intervalos de confianza al $95 \%$ y valores de $p$ de la asociación no ajustada y ajustada entre las variables de interés y la variable dicotómica construida para distinguir a las personas que tienen algún problema de uso de alcohol (AUDIT > 7, que incluye consumo de riesgo, uso perjudicial y dependencia probable) de los que no lo tienen (i.e., AUDIT $\leq 7$ ). El odds de tener un problema de alcohol en los varones fue 26 veces el odds de tener dicho problema entre las mujeres. Este hallazgo nos llevó a estratificar por el sexo de los participantes en el modelo de regresión logística múltiple.

Respecto al efecto del consumo reciente de alcohol (en las seis horas previas a la atención en la sala de emergencia), la estimación cruda mostró que tener un consumo problemático de alcohol, para quienes habían consumido recientemente dicha sustancia, fue 4,4 veces el odds de aquellos sin consumo reciente. Esta fuerza y dirección de la asociación fueron similares en el análisis multivariado (OR=5,2; IC 95\%: $2,4-11,5$; $p<0,001)$. En el caso de las mujeres no hubo asociación ni en el análisis crudo ni en el multivariado, entre uso reciente de alcohol y el uso crónico problemático de dicha sustancia.

\section{DISCUSIÓN}

El principal hallazgo de este estudio es que el ser varón y el haber consumido alcohol en las seis horas previas a la atención en una sala de emergencia se encontraron fuertemente asociados al uso problemático de alcohol,

Tabla 3: Asociación entre características clave de individuos atendidos en una de emergencia con la presencia de uso problemático crónico de alcohol.

\begin{tabular}{|c|c|c|c|c|c|c|}
\hline \multirow[t]{2}{*}{ Variable } & \multicolumn{3}{|c|}{ Asociación cruda } & \multicolumn{3}{|c|}{ Asociación ajustada* solo en varones } \\
\hline & OR & $95 \%$ IC & Valor $p$ & OR & $95 \%$ IC & Valor $\mathrm{p}$ \\
\hline \multicolumn{7}{|l|}{ Sexo } \\
\hline Femenino & 1 & & & -- & -- & -- \\
\hline Masculino & 26,4 & $9,4-74,0$ & $<0,01$ & -- & -- & -- \\
\hline \multicolumn{7}{|l|}{ Servicio } \\
\hline Medicina & 1 & -- & -- & 1 & -- & -- \\
\hline Cirugia & 2,2 & $1,3-2,6$ & $<0.01$ & 1,5 & $0,8-2,8$ & 0,20 \\
\hline \multicolumn{7}{|l|}{ Edad } \\
\hline De 14 a 18 & 1 & -- & -- & 1 & -- & -- \\
\hline De 19 a 25 & 1,3 & $0,6-3,1$ & 0,50 & 1,3 & $0,5-3,9$ & 0,60 \\
\hline De 26 a 34 & 1,1 & $0,5-2,7$ & 0,80 & 1,2 & $0,4-3,5$ & 0,73 \\
\hline De 35 a 44 & 1,2 & $0,5-3,2$ & 0,61 & 1,4 & $0,4-4,5$ & 0,61 \\
\hline De 45 a 64 & 0,6 & $0,2-1,5$ & 0,24 & 0,6 & $0,2-1,8$ & 0,35 \\
\hline 65 o mayor & 0,1 & $0,01-0,7$ & 0,03 & 0,1 & $0,01-0,6$ & 0,02 \\
\hline \multicolumn{7}{|c|}{ Alcohol en últimas 6 horas } \\
\hline No & 1 & -- & -- & 1 & -- & -- \\
\hline Sí & 4,4 & $2,5-7,7$ & $<0,01$ & 5,2 & $2,4-11,5$ & $<0,001$ \\
\hline \multicolumn{7}{|c|}{ Daño físico en pacientes atendidos en cirugía† } \\
\hline No & 1 & -- & -- & -- & -- & -- \\
\hline Sí & 2,6 & $0,8-8,0$ & 0,11 & -- & -- & -- \\
\hline
\end{tabular}

Significa que la estimación de los Odds Ratios (ORs) se realizó en un modelo que incluyó las variables servicio, edad y uso reciente de alcohol, simultáneamente.

† La variable correspondiente a daño físico esta fuertemente asociada a la variable correspondiente al servicio donde fue atendido el paciente. Esto genera colinearidad entre ambas variables, por ello solo una de ellas (i.e., servicio de atención) es tomada en cuenta en el análisis ajustado. 
el cual fue independiente de la edad, de la especialidad del servicio de atención o si la razón de atención fue por daño físico. Específicamente, nuestros hallazgos establecen que si un paciente atendido en una sala de emergencia es varón y ha consumido alcohol recientemente, independientemente de la razón de la atención y su edad, este paciente tiene aproximadamente cinco veces mayor posibilidad de tener un uso de riesgo de alcohol, un uso perjudicial o dependencia a dicha sustancia.

Algunas limitaciones y fortalezas deben ser mencionadas antes de pasar a una discusión de las implicancias de este estudio. La primera limitación tiene que ver con que este proyecto fue realizado en una sola institución de salud, lo que podría hacer que algunos científicos de la salud pública tengan dudas acerca de la validez externa y extrapolación de los resultados a otras salas de emergencia. Aunque hubiera sido ideal incluir en este proyecto a varias salas de emergencia de nuestro país, esto no fue posible por los limitados recursos con que se contó para su ejecución. Sin embargo, el hecho de que el hospital en que este estudio fue implementado sea un hospital público típico en la capital peruana, cuya área de influencia es del tamaño y características demográficas de las zonas más populosas de esta ciudad, hace confiar que los hallazgos puedan ser extrapolados a otras realidades de hospitales generales de similar magnitud, por lo menos en la ciudad de Lima. No obstante se deben realizar futuros estudios donde se incluyan otros hospitales del país para evaluar esta hipótesis.

Una segunda limitación es que hubo una participación diferenciada respecto al sexo. El hecho de que las mujeres hayan tenido mayor probabilidad de no participar en el estudio puede estar introduciendo un sesgo de selección, ya que aquellas mujeres que aceptaron participar pueden ser diferentes a aquellas que no participaron respecto a las variables de interés en este estudio. Por ejemplo, mujeres con altos niveles de consumo reciente o de consumo crónico de alcohol pueden haber escogido no participar en el estudio luego de conocer los objetivos de la investigación por el temor a ser "criticadas" o "juzgadas" por su nivel de consumo -que ellas pueden percibir en contradicción con los roles socioculturales esperados de las mujeres en una sociedad como la peruana-. Aunque se trató de asegurar la privacidad y confidencialidad de la información, es posible que no se haya podido controlar completamente el temor de aquellas mujeres de quedar en evidencia respecto a su consumo ante otras personas significativas para ellas, que pueden incluir, además de sus familiares, el personal de salud e incluso el entrevistador/a del estudio. Sin embargo, a pesar de la menor participación de las mujeres (nivel de respuesta de $74 \%$ frente a $85 \%$ de los varones), esta permaneció para ambos sexos en niveles aceptables, para lo que se espera de estudios epidemiológicos.
Una última limitación es el pequeño número de mujeres que puntuaron en el AUDIT en niveles de uso problemático de alcohol (i.e., AUDIT>7). Solo cuatro mujeres calificaron dentro de esta categoría y todas lo hicieron para uso de riesgo y ninguna para uso perjudicial o probable dependencia. Ello impidió que podamos modelar con solvencia el carácter predictivo de las variables de interés para las mujeres, lo cual puede estar explicando el por qué ninguna de dichas variables resultó asociada con el consumo problemático de alcohol en ellas (error tipo 2). Futuros estudios deben considerar diseños específicos para incrementar la participación de mujeres en el contexto de salas de emergencia. Asimismo, son necesarios posteriores estudios de validación que exploren la posibilidad de que para las mujeres los puntos de corte en el AUDIT sean menores que para lo varones, para luego valorar factores predictores de uso problemático de alcohol en ellas en el contexto de salas de emergencias.

Entre las fortalezas de este estudio tenemos el carácter epidemiológico de su diseño transversal, cubriendo siete días completos en los servicios de medicina y cirugía de una sala de emergencia promedio de Lima, usando instrumentos validados y aplicados por personal de salud capacitados para los fines de este proyecto. Además, este estudio usa datos de un proyecto realizado por nuestro grupo que constituyó, a nuestro conocimiento, el primero realizado en salas de emergencia en el Perú ${ }^{(23)}$. La validez externa de los datos de este proyecto está respaldada por sus resultados respecto al consumo reciente y crónico de alcohol y uso de drogas ilegales ${ }^{(23)}$, los cuales mostraron ser consistentes con aquellos que la bibliografía internacional reporta $(6,22,25)$. Por último, $y$ tomando en cuenta lo que hemos podido conocer en la literatura, este estudio también es el primero que específicamente señala factores predictores que ayuden a identificar personas a quienes se debería descartar problemas de uso de alcohol entre todas aquellas atendidas en salas de emergencia.

Debido a la alta proporción de personas con uso problemático de alcohol en las salas de emergencia, estas se convierten en puntos estratégicos para detectar pacientes y derivarlos a tratamientos específicos. Esta oportunidad no está siendo actualmente aprovechada, probablemente debido a la carencia de recursos y el hecho de que se asume que la labor en las salas de emergencia se debe limitar únicamente a atender el problema agudo por el cual ha llegado el paciente a dichos servicios. De aprovecharse esta oportunidad que ofrecen las salas de emergencia, impactaría directamente en aumentar el número de personas que reciben un manejo óptimo por problemas de alcohol, incluyendo intervenciones breves, 
consejería o tratamientos médicos para dependencia. Sin embargo, los limitados recursos con los que cuentan las salas de emergencia obliga a seleccionar a aquellos que realmente tienen alta probabilidad de ser un caso de uso problemático de alcohol.

El presente estudio valoró el poder predictivo para identificar casos de bebedores problema de características que el personal de salud de las salas de emergencia pueda reconocer de manera sencilla y rápida, como son el sexo, la edad, el servicio de atención, el daño físico y el uso reciente de alcohol del paciente atendido. De todas ellas, el sexo y el uso reciente de alcohol mostraron un excelente poder predictivo para detectar casos de ese tipo de problemas. Así, estas variables podrían ser usadas para seleccionar personas a las que se deba descartar (o tamizar) por un uso problemático de alcohol con instrumentos apropiados para ello (por ejemplo, usando el AUDIT).

En conclusión, debido a que los problemas por uso de alcohol constituyen una prioridad de salud en nuestro país, se deben establecer estrategias que incrementen la proporción de estos pacientes en tratamiento. La literatura apoya que se pueden usar las salas de emergencia como puntos estratégicos de identificación, manejo inicial (p.ej., intervenciones breves) o derivación (p.ej. a servicios o instituciones especializadas de tratamiento) de personas con uso problemático de alcohol ${ }^{(22-23)}$. El presente estudio señala que el aplicar un instrumento de descarte de problemas de alcohol a varones con uso reciente de alcohol que llegan a salas de emergencia ayudará a identificar de manera costo-efectiva a aquellos que requieran algún tipo de manejo para dichos problemas. En última instancia, el aumentar el número de personas en tratamiento ayudará en el corto y mediano plazo a disminuir la carga de enfermedad causada por el alcohol, ya que ello incrementa la proporción de personas en recuperación por dichos problemas, lo que impactará además en reducir la desinformación y el estigma hacia este tipo de problemas de manera gradual en la población.

\section{Fuentes de Financiamiento}

Trabajo financiado por DEVIDA y la Cooperación Técnica Belga.

\section{Conflictos de Interés}

Los autores declaran no tener conflictos de interés relacionados al presente estudio.

\section{REFERENCIAS BIBLIOGRÁFICAS}

1. Rehm J, Room R, Graham K, Monteiro M, Gmel G, Sempos CT. The relationship of average volume of alcohol consumption and patterns of drinking to burden of disease: an overview. Addiction. 2003; 98(9):1209-28.
2. Nutt DJ, King LA, Phillips LD. Drug harms in the UK: a multicriteria decision analysis. Lancet. 2010; 376(9752):1558-65

3. Lopez AD, Mathers CD, Ezzati M, Jamizon DT, Murray CJ. Global Burden of Disease and Risk Factors. Washington D.C.: The World Bank and Oxford University Press; 2006.

4. World Health Organization. World Health Report 2001Mental Health: new understanding, new hope. Geneva: World Health Organization; 2001.

5. Velásquez A. La carga de enfermedad y lesiones en el Peru y las otras prioridades del plan esencial de aseguramiento universal. Rev Peru Med Exp Salud Publica. 2009; 26(2):222-31.

6. Cherpitel CJ, Bond J, Ye Y, Borges G, Macdonald S, Giesbrecht N. A cross-national meta-analysis of alcohol and injury: data from the Emergency Room Collaborative Alcohol Analysis Project (ERCAAP). Addiction. 2003; 98(9):1277-86.

7. Cherpitel CJ, Ye Y, Bond J. Attributable risk of injury associated with alcohol use: cross-national data from the emergency room collaborative alcohol analysis project. Am J Public Health. 2005; 95(2):266-72.

8. Cherpitel CJ, Bond J, Ye Y, Borges G, Room R, Poznyak V, et al. Multi-level analysis of causal attribution of injury to alcohol and modifying effects: Data from two international emergency room projects. Drug Alcohol Depend. 2006; 82(3):258-68.

9. Cherpitel CJ. Alcohol and injuries: a review of international emergency room studies since 1995. Drug Alcohol Rev. 2007; 26(2):201-14.

10. Cherpitel CJ, Ye Y, Bond J, Borges G. The causal attribution of injury to alcohol consumption: a cross-national meta-analysis from the emergency room collaborative alcohol analysis project. Alcohol Clin Exp Res. 2003; 27(11):1805-12.

11. Borges G, Cherpitel CJ, Medina-Mora ME, Mondragon L. Violence related injuries in the emergency room: alcohol, depression, and conduct problems. Subst Use Misuse. 2004; 39(6):911-30.

12. Macdonald S, Cherpitel CJ, Borges G, Desouza A, Giesbrecht N, Stockwell T. The criteria for causation of alcohol in violent injuries based on emergency room data from six countries. Addict Behav. 2005;30(1):103-13.

13. Cherpitel CJ, Ye Y. Alcohol and Violence-Related Injuries among Emergency Room Patients in an International Perspective. J Am Psychiatr Nurses Assoc. 2010; 16(4):227-35.

14. Macdonald S, Cherpitel CJ, DeSouza A, Stockwell T, Borges G, Giesbrecht N. Variations of alcohol impairment in different types, causes and contexts of injuries: results of emergency room studies from 16 countries. Accid Anal Prev. 2006;38(6):1107-12.

15. Galvez-Buccollini JA, Paz-Soldan V, Herrera P, DeLea S, Gilman RH, Anthony JC. Links between sex-related expectations about alcohol, heavy episodic drinking and sexual risk among young men in a shantytown in Lima, Peru. Int Fam Plan Perspect. 2008;34(1):15-20.

16. Galvez-Buccollini JA, Paz-Soldan VA, Herrera PM, DeLea S, Gilman RH. Gender differences in sex-related alcohol expectancies in young adults from a peri-urban area in Lima, Peru. Rev Panam Salud Publica. 2009;25(6):499-505. 
17. Kohn R, Levav I, de Almeida JM, Vicente B, Andrade $\mathrm{L}$, Caraveo-Anduaga JJ, et al. [Mental disorders in Latin America and the Caribbean: a public health priority]. Rev Panam Salud Publica. 2005; 18(4-5):229-40.

18. Instituto Nacional de Salud Mental. Estudio epidemiológico metropolitano en salud mental 2002. Anales Salud Mental. 2002; 18(1, 2):13-81.

19. Instituto Nacional de Salud Mental. Estudio epidemiológico de salud mental en la sierra peruana 2003. Anales Salud Mental. 2003; 19(1,2):25-102.

20. Instituto Nacional de Salud Mental. Estudio epidemiológico de salud mental en la selva peruana 2004. Anales Salud Mental. 2004;21(1,2):25-98.

21. Cherpitel CJ. Emergency room and primary care services utilization and associated alcohol and drug use in the United States general population. Alcohol Alcohol. 1999; 34(4):581-9.

22. Cherpitel CJ, Ye Y. Drug use and problem drinking associated with primary care and emergency room utilization in the US general population: data from the 2005 national alcohol survey. Drug Alcohol Depend. 2008; 97(3):226-30.

23. Mazzotti G, Ordóñez C, Bustamante I, Acosta R, Contreras C, Gallo C, et al. Consumo de alcohol y otras sustancias psico-activas en pacientes atendidos en un servicio de emergencia en Lima, Peru. Revista Peruana de Drogodependencias. 2006; 4(1):115-35.

24. Monti PM, Colby SM, Barnett NP, Spirito A, Rohsenow DJ, Myers M, et al. Brief intervention for harm reduction with alcohol-positive older adolescents in a hospital emergency department. J Consult Clin Psychol. 1999;67(6):989-94.

25. Observatorio Argentino de Drogas (OAD). El uso indebido de drogas y la consulta de emergencia. Tercer estudio nacional. Tucumán: OAD; 2008.
26. Saunders JB, Aasland OG, Babor TF, de la Fuente JR, Grant M. Development of the Alcohol Use Disorders Identification Test (AUDIT): WHO Collaborative Project on Early Detection of Persons with Harmful Alcohol Consumption--II. Addiction. 1993; 88(6):791-804.

27. Rubio G, Bermejo J, Caballero MC, Santo-Domingo J. Validación de la prueba de identificación de trastornos por uso de alcohol (AUDIT) en atención primaria. Rev Clin Esp. 1998;198:11-4.

28. Bohn MJ, Babor TF, Kranzler HR. The Alcohol Use Disorders Identification Test (AUDIT): validation of a screening instrument for use in medical settings. J Stud Alcohol. 1995;56(4):423-32.

29. Aertgeerts B, Buntinx F, Ansoms S, Fevery J. Screening properties of questionnaires and laboratory tests for the detection of alcohol abuse or dependence in a general practice population. Br J Gen Pract. 2001; 51(464):206-17.

30. StataCorp. Stata statistical software: Release 10.0. College Station, TX: Stata Corporation; 2007.

Correspondencia: Fabian Fiestas, M.D., PhD(c).

Dirección: Av. Honorio Delgado 430, San Martín de Porres, Lima, Perú.

Teléfono: (+511) 985-765743.

Correo electrónico: ffiestas@epi.msu.edu

\section{Consulte la versión electrónica de la} Revista Peruana de Medicina Experimental y Salud Pública en www.pubmed.gov

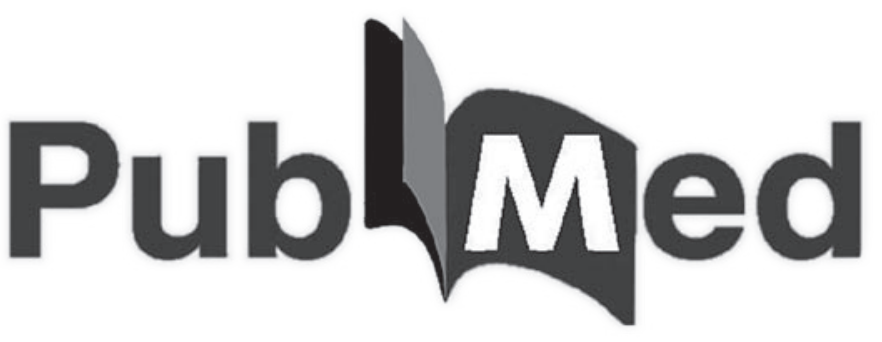

